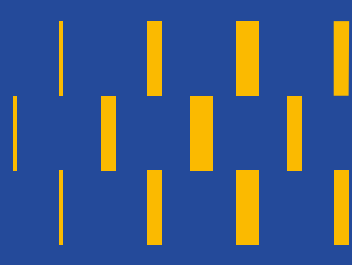

\title{
Corporalidad, emoción y discurso oral: un acercamiento a la noción del pia entre los chanés del Noroeste Argentino
}

\section{Corporality, emotion and oral discourse: an approach to the notion of pia among the Chanés of Northwest Argentina}

\author{
María Agustina Morando ${ }^{1,2,3}$ (D) https://orcid.org/0000-0002-2867-3173 \\ ${ }^{1}$ Consejo Nacional de Investigaciones Científicas y Técnicas, ARGENTINA. \\ ${ }^{2}$ Pontificia Universidad Católica Argentina, Instituto de Investigaciones, Facultad de Ciencias Sociales, \\ ARGENTINA. Email: agustinamorando@uca.edu.ar \\ ${ }^{3}$ Centro de Investigaciones Históricas y Antropológicas, BOLIVIA.
}

\section{Resumen}

El objetivo de este artículo es realizar un acercamiento a las representaciones verbales de lo emocional y las formas en las que estas se expresan entre los chanés del Noroeste Argentino. La expresión de la emoción entre los chanés pasa mayormente por el pia, un órgano que actúa como locus por antonomasia de la expresión de los estados anímicos de una persona. Teniendo esto en cuenta, el foco estará puesto en los registros orales y las experiencias de la vida cotidiana chané a partir de las cuales se manifiestan las emociones y sus significados culturales. Este trabajo pretende ser un aporte al estudio de la construcción social del cuerpo y la concepción de persona desde la óptica de la emoción verbalizada.

Palabras clave: emoción y corporalidad, grupos indígenas sudamericanos, Gran Chaco.

\begin{abstract}
The aim of this paper is to approach the representations about the verbal ways in which the Chanés of Northwest Argentina express emotions. The expression of the emotion is mainly associated with $p \dot{t a}$, an organ that acts as a locus par excellence of the expression of the states of mind of a person. Bearing this in mind, the focus here will be on the description the contexts and experiences of everyday Chané life in which emotions and its significance are culturally manifested. This work aims to contribute to the study of the social construction of the body and the conception of the person from the perspective of the verbal emotion.
\end{abstract}

Keywords: emotion and corporality, South American indigenous groups, Great Chaco.

Recibido: 9 julio 2020. Aceptado: 30 noviembre 2020 


\section{Introducción}

De acuerdo con los resultados arrojados por la Encuesta Complementaria de Pueblos Indígenas de 2004-2005 (INDEC 2004/2005), aproximadamente 2100 personas se reconocen como "chanés" en la República Argentina. La mayor parte se concentra en cuatro poblados del departamento San Martín de la provincia de Salta. Los chanés son un grupo de origen arawak que, tras una serie de procesos migratorios sobre el piedemonte andino, atravesaron a partir del siglo XVI un proceso de "guaranización" a manos de grupos de origen tupí-guaraní que los dominaron militar y políticamente, dando origen a una "civilización mixta" o "sociedad mestiza" conocida en la etnohistoria del Chaco como "chiriguano" (Métraux, 1948; Magrassi, 1968; Susnik, 1968; Rocca, 1973; Combès y Saignes, 1995; Saignes, 2007). Debido a su mestizaje, los chanés comenzaron paulatinamente a adoptar la lengua y muchos rasgos culturales de origen guaraní (Combès, 2007) y, por esta misma razón, hablan actualmente una variante del llamado "guaraní chaqueño" o "chiriguano" (Dietrich, 1986; Aikhenvald, 1999; Gustafson, 2014). Ahora bien, la integración chané dentro del conjunto chiriguano no debe ser entendida como una asimilación total, pues la herencia arawak es perceptible en algunos aspectos de la cultura material, ciertos rasgos de la organización sociopolítica e incluso en ciertos marcadores lingüísticos (Combès y Villar, 2004; Villar, 2006; Morando, 2015). Esto explica el hecho de que, a través de la historia, los chanés hayan mantenido una conciencia clara de su diferencia étnica, más puntualmente todavía en la cuenca del río Itiyuro y en algunos sectores del Isoso (Combès, 2005; Villar, 2006; Bossert, Combès y Villar, 2008).

El objetivo de este trabajo es describir etnográficamente las representaciones sobre lo emocional y las formas en las que estas se expresan verbalmente entre los chanés del Noroeste Argentino (NOA). Entre los chanés, la expresión de la emoción se asocia mayormente con un órgano llamado pía que opera como el centro de la expresión de los estados anímicos de una persona. A fin de profundizar en la comprensión de ello, describiré aquí una configuración semántica-cultural expresada en unidades léxicas, palabras, frases o expresiones que son vehículo de representaciones más generales acerca de la expresión de lo emocional y lo corporal. La posibilidad de analizar el lenguaje de las emociones deja entrever a la vez aspectos singulares relacionados con la experiencia social y, asimismo, con la construcción de la persona y sus "estados anímicos" (Descola, 1996 [1987]; Surrallés, 2009 [2003]). Se trata, por lo tanto, de un intento analítico de describir las formas lingüísticas y culturales que asume la emoción para este grupo indígena particular, y a la vez de profundizar, a partir de datos empíricos recogidos durante mi trabajo de campo, el estudio de algunas dinámicas de la producción de la subjetividad. En definitiva, tomando como base analítica la categoría nativa pía propongo poner de relieve la importancia de considerar el discurso indígena sobre el cuerpo y sobre la persona como generador de praxis social (Seeger, Da Matta y Viveiros de Castro, 1979).

Para lograr ese objetivo, el análisis aquí propuesto retoma dos elementos -la etnografía y el habla- que permitirán abordar de modo más completo el dominio de la expresión discursiva de la emoción. Me valdré para ello de ciertos aportes teóricos vinculados con la llamada etnografía del habla, que proporcionan un acercamiento más antropológico al discurso, concebido no solo como mera trama textual sino a la vez como fenómeno verbal perteneciente al nivel de la lengua en su contexto de uso (Bauman, 1975, 1977, 1986; Hymes, 1981; Gumperz, 1982; Sherzer, 1987; Bauman y Briggs, 1990). Esto habilita, entonces, partir descriptivamente desde lo discursivo como locus privilegiado de los procesos de producción cultural (Golluscio, $2019[2002])$. 
El fin ulterior de este artículo es contribuir a los estudios sobre emoción y corporalidad entre grupos indígenas sudamericanos, problemática que ha comenzado a visibilizarse en el horizonte de los estudios americanistas fundamentalmente a partir de las décadas de 1960 y 1970. Desde esta óptica, el cuerpo es concebido como una entidad que condensa componentes fisiológicos, pero además anímicos y sociales, a partir de los cuales es posible construir, asociar y/o rastrear una serie compleja de significados. ${ }^{1}$

\section{Algunos aspectos acerca de la construcción de la persona chané}

Antes de proceder con el tema principal de este trabajo, es preciso desarrollar brevemente algunas cuestiones relevantes sobre la construcción de la persona chané. Para comenzar, la concepción chané de persona tiene origen en tres entidades principales llamadas teko, ä y tete. En primer lugar, teko es un concepto de una notable polisemia que no solo es traducido por los chanés como 'vida' sino también como 'salud', 'costumbre', 'actitud' o 'modo de vivir', tanto desde una perspectiva positiva como negativa. Así, por ejemplo: chereko maëpora, ikavi 'mi modo de vivir o costumbre es bonito, bueno'; ñandereko kuarai 'nuestro modo de vivir o costumbre es asî'; jeko ambuae 'su modo de vivir o costumbre es diferente'.

Debido al establecimiento en el Chaco de misiones jesuitas y luego franciscanas durante el período comprendido entre los siglos XVII y XX (Langer, 1995), los chanés -así como otros grupos étnicos guaraníhablantes del Chaco occidental (avas, isoseños, simbas)- atravesaron por un largo proceso de misionalización y de contacto con el frente colonizador. En el caso específico de los chanés del Noroeste Argentino entre quienes llevé a cabo esta investigación, este proceso ha sido más reciente -desde mediados del siglo XX- y tal vez no tan sistemático como en el caso del Chaco boliviano. Sin embargo, como consecuencia de ello, lo que sí es cierto es que una gran cantidad de conceptos relacionados con la cosmología indígena sufrieron una serie de transformaciones que permitieron incorporar lo cristiano a la vida indígena (Villar, 2008; Morando, 2017). Teko es uno de los tantos ejemplos de ello ya que, a partir del mismo, se ha encontrado un "equivalente" en la lengua indígena a las ideas cristianas de "virtud' (chereko kavi, literalmente 'mi modo de vivir, mi costumbre buenos') y 'pecado' (chereko pochi, literalmente 'mi modo de vivir o mi costumbre malos'). ${ }^{2}$

Ahora bien, este concepto no solo es relevante para las personas sino, también, para los animales y las plantas (Villar, 2005, p. 79). En efecto, es preciso tener en cuenta que muchos de los conceptos a los que referiré aquí también pueden asociarse con animales, plantas y seres espirituales, extendiendo de esta forma el horizonte de la "humanidad" (Leenhardt, 1997 [1947]).

Otro de los conceptos importantes para entender la dinámica de la construcción de la persona y su principio vital entre los chanés es el $\ddot{a}$. El $\ddot{a}$ es lo que, de forma preliminar, podría llamarse 'componente espiritual' de la persona. Este puede ser entendido como 'sombra' en el sentido más estricto del término: sea de un ser vivo o de un objeto (Villar, 2008, pp. 344-345). No es

1 La bibliografía al respecto es virtualmente inabarcable, pero pueden destacarse fundamentalmente los aportes respectivos de Da Matta (1976), Overing (1977), Seeger, Da Matta y Viveiros de Castro (1979); Descola (1996 [1987]), Viveiros de Castro (1996), Surrallés (2009 [2003]), Palmer (2005), Vilaça (2005), Córdoba (2006, 2008), Tola (2007), Gómez (2010) y Montani (2012).

2 Debido al carácter inalienable de los nombres de las partes del cuerpo y los términos de parentesco, éstos aparecen siempre acompañados por marcas de persona que indican un poseedor: $1^{\circ}$ persona singular che-, $2^{\circ}$ persona singular $n d e-/ n e-, 3^{\circ}$ persona singular $i$-liy-liñ-/j-, $1^{\circ}$ persona plural inclusiva ñande-/ ñane-, $1^{\circ}$ persona plural exclusiva $n$ dore-, $2^{\circ}$ persona plural pe-/pende-, $3^{\circ}$ persona plural $i \ldots(-$ reta $)$. 
casual, entonces, que este mismo término sea utilizado además como neologismo para referir a una 'imagen', 'ilustración' o 'fotografía'. Por eso, cuando los chanés distinguen a una persona en un video o una fotografía, suelen decir que el $\ddot{a}$ de una persona está allí y que lo están viendo: nde ä ö̈ 'está tu $\ddot{a}$; iä jokope 'el $\ddot{a}$ imagen de él/ella está alli’'; che aekita nde $\ddot{a}$ 'yo sacaré tu $\ddot{a}$, es decir, 'yo te tomaré una foto'; iä ueki 'yo le saco su $\ddot{a}$ a él/ella', es decir, 'yo le tomo una foto a él/ella'. El ä queda entonces asociado con las ideas de 'sombra', 'imagen', 'representación' o 'proyección'; con algo que, en definitiva, parece ser más captable, observable. La idea de ä está estrechamente vinculada a su vez con otra noción fuertemente arraigada en las creencias escatológicas, que es la de aña. Muchos chanés afirman que aña sería un estado ulterior del $\ddot{a}$ pero que no es, sin embargo, cualquier $\ddot{a}$, sino el de aquellas personas que han padecido una muerte violenta o trágica (por ejemplo, por homicidio o suicidio), o bien un tipo de muerto que guarda una cierta distancia con la memoria de los vivos; así, mientras que al ä se le reconoce una cierta identidad relacionada con alguna persona fallecida, el aña se presenta como una suerte de figura "despersonalizada" (Métraux, 1930; Villar, 2005, 2011; Villar y Bossert, 2014).³

De hecho, durante la celebración anual del arete guasu ('gran tiempo verdadero') o 'carnaval', hacen su aparición unas máscaras que los chanés llaman aña jäti (literalmente, 'cuerno del aña') (Palavecino, 1949; Rocca y Newbery, 1972; Rocca, 1973; Villar y Bossert, 2014). De acuerdo con Villar y Bossert (2014), los aña solamente pueden circular en el mundo de las personas durante los últimos días de esta celebración ritual, que se extiende aproximadamente entre enero y abril; el resto del ańo los hablantes entienden que 'habitan' en el tronco del palo borracho o yuchán (Chorisia insignis). Las máscaras de hecho se confeccionan con la madera de ese árbol y el proceso debe realizarse en secreto, en lo posible en el monte. En el trascurso de la celebración del arete guasu, los enmascarados se entremezclan entre los participantes bailando, bebiendo y haciendo chanzas; de esta forma, las máscaras funcionarían como una suerte de "puente" mediador momentáneo pero efectivo entre las temporalidades del mundo de los vivos y de los muertos (Villar y Bossert, 2014).

$\ddot{A}$ y aña son también un interesante ejemplo de las formas en las que la doctrina cristiana se ha escurrido en la lengua y la vida indígenas. Tomaré, para ejemplificarlo, unos fragmentos de una carta escrita por el misionero franciscano Doroteo Giannecchini, quien durante toda la segunda mitad del siglo XIX trabajó en distintas misiones chiriguanas y chanés. El religioso expresa de forma clara, en una misiva escrita en 1869 al Discretorio del Colegio de Propaganda Fide de Nuestra Señora de los Ángeles, una cierta vacilación sobre el uso de los términos aña y $\ddot{a}$ en un catecismo escrito por el franciscano Alejandro Corrado que saldría publicado en 1871 (Corrado, 1871). Respecto del vocablo aña, Giannecchini observa los sentidos asociados al vocablo incluyendo aquellos que son más cercanos a lo cristiano y aquellos otros más cercanos a lo indígena:

$7^{\circ} A \tilde{n} a$. Demonio. [...] Aña es todo lo que tiene aspecto de feo y de mal. Aña son los finados, a los bultos que ven de noche reales o ideales que sean; los sueños, las pesadillas, o mejor sus efectos; toda larva o espectro feo y asustador; Aña son los brujos malos: que decirle a uno que es un pícaro, un perjudicial, un matador y

3 Compárese esto, por ejemplo, con el caso del ahot entre los wichís, vecinos de los chanés en el Noroeste Argentino. De acuerdo con Palmer (2005) y Montani (2012), al fallecer una persona se transforma en un espíritu maligno. Para evitar los eventuales daños que puede producir un ahot, los wichís siguen un ritual funerario que implica deshacerse de todos los bienes materiales que poseía el fallecido en vida rompiendo con cualquier lazo social que los pueda vincular con aquel espectro. 
cosa semejante, le dicen que tiene consigo hartos Añas. Aña! Aña! Aña! dicen las madres a sus hijos para asustarlos; en el mismísimo sentido que en Toscana les dicen la Befana! Befana! el brujo! el brujo! el brujo! el tata! El espíritu misterioso! (Giannecchini, 1869, f. 2).

Lo mismo sucede con la descripción que realiza sobre el $\ddot{a}$ (aquí yonêa $d^{4}$ :

90. Yonea-Alma. Término mucho más equívoco, oscuro e incierto [...] Yonêa, sombra [...]. Yonêa, nuestro reflejo producido por un espejo, cristal, vaso, agua, etc. Yonêa, nuestra imaginación en el sueño. Yonêa, visión en durmiendo [...]. Todo efecto de pesadillas, calenturas, delirios, etc., lo expresan con Yonêa; y no teniendo en su bárbaro discurso como expresar estos fenómenos del sueño, delirio, calenturas, dicen disparates respecto a esta Yonêa. de ahí es que después de la muerte, la Yonêa se vuelve Aña. Se revela a uno y otros, que la ven andar lo andado, quejarse y cosas semejantes; y que después de haber cumplido con todas las vagaciones y trasmigraciones de ellos, esta sombra, esta Yonêa, se va al pueblo del Aña y al cabo muere del todo [...] (Giannecchini, 1869, f. 2).

Por otra parte, la idea del "cuerpo" como soporte material y biológico de la persona está relacionado fundamentalmente con la idea de tete. Esto también es aplicable a los animales: así, por ejemplo, se dice cherete michi 'mi cuerpo es pequeño', 'tengo un cuerpo pequeño'; jete täta 'su cuerpo es fuerte', 'tiene un cuerpo fuerte'; pero: guasu jete 'el cuerpo de la corzuela'; taitetu jete 'el cuerpo del chancho de monte'. Alternativamente, esta palabra puede emplearse para referir al 'tronco' y al 'tallo' de una planta (Villar, 2005, p. 29, 2011, p. 175). Brevemente, puede decirse que la generalidad de los órganos internos que componen el tete humano lleva el nombre churi, mbaechuri o churi-churi, que los chanés traducen al castellano como 'tripas' o todo aquello que abarcaría a los 'intestinos' y a las 'vísceras'. Además, distinguen los nombres de una serie definida de órganos. Por ejemplo, el 'cerebro' lleva el nombre de ñaputuu. En el caso de las mujeres, el 'útero' recibe el nombre de membiriru; término compuesto por membi 'hijo o hija de la mujer' y riru 'contenedor' o 'recipiente', significando literalmente el 'contenedor o recipiente del hijo o hija'. Existe, por otro lado, un conjunto de órganos entre los que se encuentran el 'corazón', el 'estómago', los 'pulmones' y el 'hígado' que reciben conjuntamente el nombre de pia. Este último, como ya he adelantado, desempeña un papel fundamental en la expresión de la emoción y la producción de la subjetividad.

\section{El pia como el centro de expresión emocional}

$\mathrm{Al}$ indagar sobre las formas en las que aparece el pia en el discurso cotidiano chané se observa una serie de matices que van desde lo más literal hasta lo más metafórico vinculados, por un lado, a la expresión de los procesos fisiológicos y orgánicos del cuerpo humano y, por el otro, a la manifestación de las emociones y las conductas. Ambas facetas merecen ser analizadas de forma conjunta para poder lograr una mejor comprensión de la forma en la que este órgano es concebido por los chanés. Esta exploración puede realizarse a través de la lengua (puntualmente a partir de una serie de expresiones que se repiten continuamente en el habla del día a día)

4 En los ejemplos tomados de fuentes que no provengan de mi propio trabajo de campo, se respetará la grafía original.

5 Esto puede compararse con el concepto de - hi o 'recipiente' wichí estudiado detalladamente por Montani (2017, p. 209), en el que el 'útero' o lëkahi se traduce como 'recipiente no propio'. 
lo que puede permitir entrar más en detalle sobre aquello que en el discurso cotidiano se "da por sentado" o se sobreentiende sobre el pia.

En primer lugar, el pia cumple un papel crucial a la hora de expresar algunas dolencias y malestares físicos. Por ejemplo, los 'malestares estomacales' suelen marcarse mediante locuciones como chepia jasi 'mi pia duele'. Los 'estados de acidez estomacal' se expresan mediante frases como chepia raku 'mi pia está caliente'. ${ }^{6}$ Además, es común escuchar nombrar los 'estados convulsivos' como chepia riri, literalmente 'mi pia se sacude'. Otra frase que suele escucharse es chepia ruru, en la que ruru significa 'hinchado'. Esta hinchazón se relaciona generalmente con infecciones parasitarias intestinales, uno de cuyos síntomas más visibles es la inflamación del área abdominal. Puede sumarse a esta lista la palabra empleada para referir a la 'pirosis' o 'acidez estomacal': chepia ratapi. Tatapi significa literalmente 'carbón', lo cual tal vez pueda relacionarse con la sensación de quemazón que esto produce. Por último, las 'náuseas' son llamadas chepiaro, donde iro significa 'ácido' o 'amargo', acaso relacionando la dolencia con el reflujo gástrico producido con anterioridad al vómito. Los 'problemas cardíacos' también suelen evocarse por medio del vocablo pia. Por ejemplo, chepia rirt 'mi pia se sacude' se utiliza no solo para nombrar los estados convulsivos sino además para designar la 'taquicardia' o las 'arritmias cardíacas'.

Ahora bien, otras referencias al pia pueden encontrarse en un sinfín de expresiones relacionadas de un modo mucho más amplio con lo emocional y lo conductual. Así, la locución ipia guasu, literalmente 'mi $p \dot{t} a$ es grande', se utiliza para referir a una persona 'testaruda', 'engreída', 'arrogante' o 'soberbia'. A fin de enfatizar aún más la idea puede decirse ipia guasu avasa, literalmente 'su pia se pasa de grande'. Tomaré a continuación para ilustrar esto, un fragmento de un relato autobiográfico registrado por mí en 2019 de Korösai, un varón chané de 69 años. En él el narrador cuenta algunos episodios relacionados con su infancia y su juventud y, en el fragmento seleccionado, relata puntualmente la dificultad que tenía de expresarse en castellano cuando era niño utilizando esta locución para explicar precisamente la actitud que los niños adoptaban frente al idioma castellano:

[...] Cheru amiripe jae: "AIKUAA KARAINEEE CHERU::/ ROIKUAA KUATIA::/ NDORE ROIKUAA::" $\rightarrow / /$

"CHESH::/ che aikuaal Aikuaa

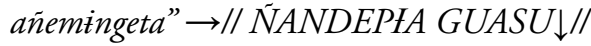

\section{$J A M A /$ ñande estudia $\uparrow / /$}

Hasta ahora entiendo algo de castellano $\rightarrow / /[\ldots]$

\author{
[...] Le decía yo a mi finado padre: \\ "NO SÉ LA LENGUA DE LOS \\ CRIOLLOS PADRE MÍO::/ NO \\ SABEMOS ESCRIBIR::/ \\ NOSOTROS NO SABEMOS::" $\rightarrow / /$ \\ "MADRE MÍA::/ no sé/ no sé hablar" $\rightarrow / /$ \\ NUESTROS PIA ERAN \\ TESTARUDOS $\downarrow / /$ \\ PERO/ estudiábamos $\uparrow / /$ \\ Hasta ahora entiendo algo de \\ castellano $\rightarrow / /[\ldots]^{7}$
}

6 Vinculadas con el malestar físico y la dolencia, las categorías asociadas con lo frío y lo caliente son un tema recurrente en las culturas sudamericanas. Sólo por citar un ejemplo, me remito una vez más al cercano caso wichí. En su estudio sobre el husék o 'buena voluntad', John Palmer (2005, p. 199) describe una serie de metáforas relacionadas con lo frío y lo caliente; por ejemplo, en el uso de 'palabras frescas' o techaj zlamet para resolver los conflictos contrapuestos con el pensamiento asocial, más bien vinculado con lo caliente.

7 Para la transcripción en lengua indígena utilicé las siguientes marcas o convenciones tomadas del sistema Jefferson: entonación ( $\uparrow$ entonación ascendente, $\downarrow$ entonación descendente, $\rightarrow$ entonación sostenida), volumen (MAYÚSCULA aumento de volumen, ${ }^{\circ}$ disminución de volumen), pausas (/ pausa corta, 
Es importante señalar que en torno de esta expresión surge una serie de dificultades exegéticas. Se debe tener en cuenta que, debido a la larga historia de misionalización por la que los chanés han atravesado, la mayoría de los hablantes es 'católica' o 'evangelista'. En estos contextos, esta expresión suele tomarse en el sentido de la casuística cristiana y puede designar también a la 'bondad', la 'generosidad' o la 'rectitud' de una persona.

En otros casos, como el de la expresión iptatäta 'su pia es duro', se utiliza para caracterizar a una persona que no puede establecer una relación de empatía con los otros. Por el contrario, ipiatätaa 'su pia no es duro' refiere a una persona 'comprensiva' y 'compasiva'. De forma similar, ipia ikai -literalmente, 'su pia está bien'- se emplea para referir a aquellas personas que ostentan una cierta 'bondad', 'amabilidad', 'rectitud' e incluso 'honestidad'. Su opuesto, ipia ikavia 'su pia no está bien' puede significar lo contrario a la expresión anterior, pero también puede referir a una persona que se encuentra en un estado de 'indecisión', 'inseguridad' o 'indeterminación'.

En otros casos, como por ejemplo chepia ndere, cuya traducción literal sería 'mi pia es por vos', los hablantes señalan la 'preocupación', 'atención' e incluso el 'cariño' de una persona hacia otra. Lo mismo sucede con expresiones como ndikua che pia pe nei, literalmente 'vos sabés que estás en mi pia', o simplemente che pia pe nei, literalmente, 'estás en mi pia'. Suele decirse esto a una persona que se recuerda, especialmente si se encuentra lejos. Se suma otra acepción con un significado más o menos análogo como che piarai mi jeseño, literalmente 'mi pia es pequeño solo por ti', que suele dirigirse a alguna persona por la cual se siente una gran 'estima' o 'apego'. Tomaré a continuación un ejemplo concreto de uso de esta locución en un canto de cortejo masculino que registré en 2017, cantado por un hombre llamado Köko, en ese momento de 67 años, mientras recordaba de qué modo halagaba en su juventud a quien luego se convertiría en su esposa. Nótese, aquí, la forma en que la expresión es acompañada por el uso de ciertos recursos discursivos empleados para reforzar el significado (Henry, 1936): un aumento del volumen de la voz, una entonación ascendente y separada por una pausa larga del resto del verso. Estos recursos logran, sin duda, reforzar la intencionalidad del significado:

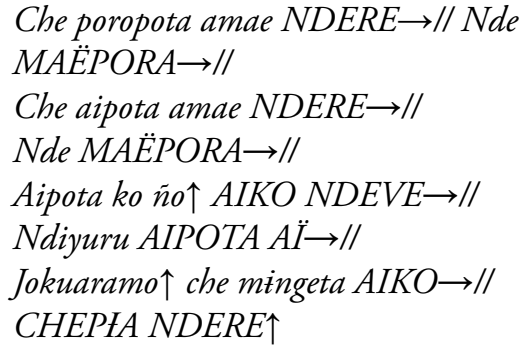

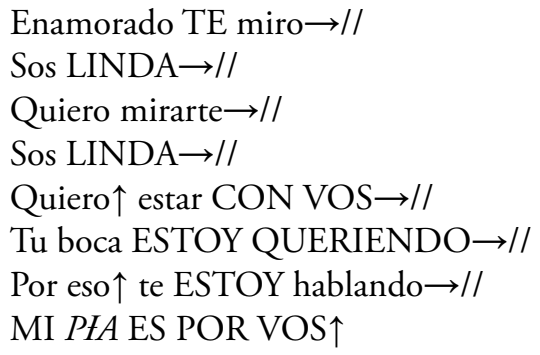

En cambio, en los momentos de aflicción, tristeza y angustia, es muy común escuchar que los hablantes digan chepia utiti, literalmente 'mi pia tiembla'. En tales situaciones el pia puede incluso llorar: che pia uyao, 'mi pia llora'. Cuando una persona siente miedo, también es común escuchar la expresión chepia kiye, literalmente, 'mi pia tiene miedo'. El 'estupor' y el 'asombro', por su parte, pueden expresarse mediante la locución chepia mano; es decir, 'mi pía muere', claramente en sentido figurado.

// pausa larga), velocidad (<> emisiones rápidas, $><$ emisiones lentas), duración (:: duplicación o triplicación de vocales y consonantes), otros ( ( ) acciones no verbales, [...] pasaje inaudible / omisión). 
Ciertos estados emocionales agitados pueden expresarse diciendo chepia imbokere, lo cual es traducido por los chanés como 'mi pia está loco'. Aquí, nuevamente, la traducción puede ser amplia. La locución puede utilizarse para describir a una persona que se encuentra ocasionalmente desorientada, o en cierta forma aturdida, e incluso para expresar estados graves de insania mental. Un sinónimo de ello podría ser además ipia ikavia 'su pia no está bien'. Así, tomo el ejemplo de un mborakua o 'consejo' relevado en 2018 y pronunciado por un muchacho de 26 años llamado Alejandro hacia su hermana menor, Roxana, de 19 años, quien se encontraba algo más callada que de costumbre durante los últimos días, preocupada por un asunto personal: aguiye jeta ñemingeta ye. Ndepia ikaviata: 'No pienses mucho. A tu pia le hará no estar bien'. El 'enfado' y el 'hastío' pueden designarse en chané mediante la expresión ipia pochi 'su pía está enfadado'. Los momentos de 'serenidad', 'calma' y 'sosiego' pueden marcarse apelando a la expresión chepia rö̈sa, literalmente 'mi pia está frío'. Un equivalente de ello podría ser chepia vevii, es decir, 'mi pia es liviano': jae joke ipta vevii guivepe 'él/ella duerme con su pia liviano', es decir, 'duerme tranquilamente'. La 'mezquindad' y la 'avaricia', por otro lado, pueden señalarse con las expresiones ipía ñemboete o ipia jakatëi 'su pia es mezquino'.

Es importante señalar que, al igual que muchos otros conceptos que pertenecen al ámbito del cuerpo humano, pia también puede aplicarse a los animales. Así, puede decirse, por ejemplo, yagua pia 'el pia del perro'; guasu pia 'el pia de la corzuela'; ayuru pia 'el pia del loro'. Sin embargo, la diferencia entre los animales y los humanos parece radicar en que en los primeros el sentido del pia no puede extenderse más allá de lo orgánico.

Ahora bien, si me remitiera a las obras de registro disponibles para esta lengua, podría observarse la misma ambigüedad. Por ejemplo, en el caso del Diccionario Breve Chiriguanae del franciscano Pedro León de Santiago (1791) la palabra pia aparece traducida como 'barriga', 'estómago' o 'corazón'. Así, para el primer caso, el religioso escribe chepuia jaci 'duéleme la barriga' (1791: 143); puia acu 'el calor natural de la barriga' (1791, p. 143); che mopiatal che mopia ata che rembiu 'lo que comí me ha endurecido el estómago' (1791, p. 143). Para el caso de las traducciones que refieren al 'corazón', León de Santiago cita lo siguiente: puiangeco 'aflicción de corazón' (1791, p. 143); ipuia miri 'corazón chico, nada sufrido, que luego se enfada' (1791, p. 143); che puia ñopu nde reco aci rechaca 'me lastima el corazón verte así enfermo' (1791, p. 143); puiaro 'corazón amargo' (1791, p. 143); puia cataupiri 'buen corazón' (1791, p. 143).

Casi un siglo después, el mencionado Giannecchini (1916 [1896]) en su Diccionario chiriguano-españollespañol-chiriguano vuelve a incurrir en esa plasticidad semántica. En la entrada que refiere a $\not \dot{t} a$, nuevamente se observa que se define de un modo muy similar al que se lo define en otras fuentes: 'estómago', 'corazón', 'entrañas' (1916 [1896], p. 171). A continuación, Giannecchini completa una lista extensa de expresiones que refiere al pia como 'corazón' de los cuales retomaré solamente algunos aquí: piamarangatu, piamarantu 'tener corazón magnánimo, bueno, noble' (1916 [1896], p. 172); piamaya 'tener corazón contento, sosegado, consolado, satisfecho' (1916 [1896], p. 172); pia ñiñi 'latido de corazón, potencia intelectiva' (1916 [1896], p. 172); piachí, piachii 'latir fuerte el corazón, palpitar' (1916 [1896], p. 172).

Sin embargo, es importante remarcar que muchas de estas expresiones referidas a lo emocional pueden utilizarse sin necesidad de recurrir compulsivamente al concepto de $p \dot{t a}$. Por ejemplo, es común escuchar que los chanés digan ne rakatëi en lugar de nepia jakatëi para decir 'sos mezquino', o che pochi en lugar de chepia pochi para marcar que uno se encuentra 'enfadado'; o simplemente imbokere en lugar de ipia imbokere para referirse a una persona que está emocionalmente agitada. Cuando se indaga acerca de cuáles son las diferencias en el uso de las 
expresiones en las que aparece el concepto del pia y aquellas otras en las que no, los hablantes argumentan que en el primer caso se trataría de algo más "interior" o que "sale directamente de dentro del cuerpo". Así, en el universo discursivo chané todo parecería sugerir que las expresiones que incluyen este concepto poseen una mayor potencia semántica. ${ }^{8}$

Ahora bien, algunos chanés, reconocen asimismo como categorías específicas para nombrar exclusivamente al 'corazón' en tanto órgano, el vocablo songo, préstamo del quechua sonqo que coexiste con un sinónimo proveniente de la propia lengua de los chanés que es chörokoko, y cuyo uso es bastante marginal. Me interesa profundizar puntualmente sobre el quechuismo songo; no se trata, en efecto, de una palabra demasiado extendida entre los chanés, pero hay hablantes que hoy en día la utilizan. Lo curioso sobre ella es que, a pesar de ser utilizada entre ellos para referir exclusivamente al 'corazón', Jürgen Golte (1973) explica que en quechua el concepto es mucho más complejo y parece, de hecho, alcanzar una complejidad equivalente a la del pia entre los chanés. A partir de un estudio similar al realizado aquí, en el que analiza diferentes fuentes lingüísticas del siglo XVI en quechua, el autor explica que -más allá de la traducción inmediata de sonqo como 'corazón'- el concepto esconde más en su nutrida semántica. En efecto, se emplea también para denominar las 'entrañas', en especial el 'corazón' y el 'estómago'. Al igual que en el caso del pia, el sonqo puede variar en su tamaño, forma y temperatura, indicando cambios en los estados emocionales: puede ser grande si la persona es 'briosa' o 'animosa', pequeño si la persona es 'tímida', liviano si la persona es 'inhábil' o 'necia', caliente si la persona siente 'ira' o 'enojo', o bien frío si la persona está 'inmóvil' (Golte, 1973, pp. 213214). Esto resulta interesante ya que los chanés justamente han habitado históricamente una zona de transición de gran importancia entre las tierras altas y las tierras bajas, con lo cual la influencia de lo andino es imposible de desestimar.

Empero, a pesar del hecho de que la mayor parte de la manifestación emocional se canaliza por el $p \dot{a} a$, lo cierto es que también existe un conjunto de emociones que existen independientemente de ese canal expresivo. Por ello, a continuación, revisaré otro repertorio léxico relacionado con la expresión de lo emocional que coexiste con el registrado. Comenzaré por el concepto de parareko, que los chanés traducen al castellano como 'compasión' o 'pena'. Es común, así, escuchar entre los hablantes frases como: che aiparareko 'tengo pena'; ndore ndui parareko 'nosotros tenemos pena de ti'; aiparareko oï vaera 'tengo pena de cómo anda'.

Otra palabra de uso corriente es piriau, que los hablantes relacionan con la 'desgracia' o la 'miseria'. En este sentido, pueden advertirse con frecuencia en el discurso cotidiano expresiones como: tuicha ipere piriau 'qué desgraciado/a está lastimado/a'; iyengu piriau 'qué desgraciado/a no puede hablar'; ipiru piriau 'qué desgraciado está flaco/a'. Cuando existe la sospecha de que una persona finge sobre algo, suele decirse jae ipiriau iyeyapo 'se hace el/la desgraciado/a'.

8 Para referencias comparables en otros grupos indígenas chaqueños, puede mencionarse el lquiyaqte toba (Tola, 2007). Cúneo y Messineo (inédito) registran distintas expresiones análogas a las registradas entre los chanés: aso María no?on da lkizaqteyaGak ('el corazón de María es bueno'), noyiitak añi lkizaqte (literalmente, 'su corazón llora', i.e. 'está triste'), etc. Sobre el caso wichí, puede referirse nuevamente al trabajo de Palmer (2005) sobre la 'voluntad' o husék, que se encuentra fundamentalmente en el 'corazón' o t'otle, órgano que imprime un cierto 'verdor' o watsancheyaj lewuk a la vida de la persona. Geográficamente más distante, cuestiones similares han sido registradas respecto del shinana entre los chácobos de Bolivia (Córdoba, 2006), el del magish entre los candoshi de Perú (Surrallés, 2009 [2003]) o el del inintai entre los achuar de Ecuador (Descola, 1996 [1987]). 
Otro de los vocablos utilizados para reflejar algo digno de pesar o que causa dolor es jasikatu que se compone de jast 'dolor', 'pena' y el morfema katu 'mucho', bastante'; por lo tanto, podría traducirse como 'mucha pena', 'mucho dolor'. Ahora bien, si jasi se encuentra generalmente como referencia al dolor físico (como en el caso de chäka jasi 'dolor de cabeza'; chepisa rasi 'dolor de oído'; etc.), en esta expresión particular está más bien relacionado con lo emocional, tal como se advierte en los siguientes ejemplos: jasikatu aiko 'ando con mucha pena'; jasikatu piriau uiko 'anda desgraciado con mucha pena'; jasikatu chesil cheru 'mucha pena por mi madre/padre'; jasikatu chemembi 'mucha pena por mi hijo/a' (dice la mujer); oiko jaeño, jasikatu 'anda solo/a, qué pena'. Tomaré para ilustrar mejor esto un fragmento discursivo de un relato que recopilé en 2018 en el que una mujer de 85 ańos, llamada Guadalupe, recuerda y relata algunos hechos de su pasado en ocasión del fallecimiento reciente de un familiar:

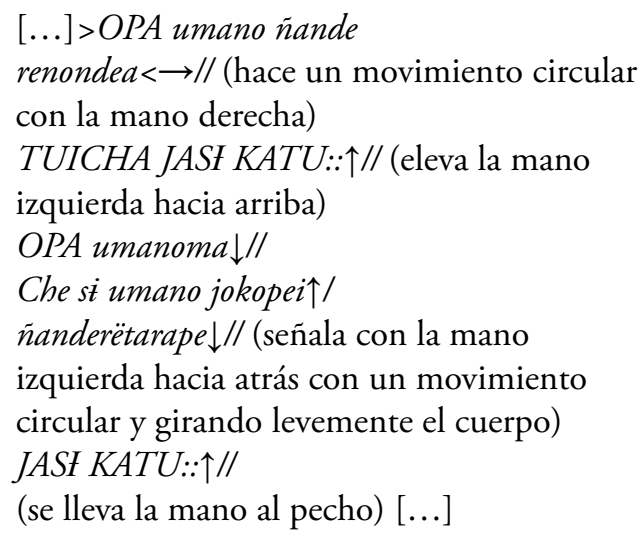

$[\ldots]>O P A$ umano ñande renondea $<\rightarrow / /$ (hace un movimiento circular con la mano derecha)

TUICHA JASI KATU::^// (eleva la mano izquierda hacia arriba)

OPA umanoma $\downarrow / /$

Che si umano jokopei $\uparrow /$ ñanderëtarape $\downarrow / /$ (señala con la mano izquierda hacia atrás con un movimiento circular y girando levemente el cuerpo) JASH KATU::^//

(se lleva la mano al pecho) [...]

$[\ldots]>$ TODOS nuestros antepasados ya
murieron $<\rightarrow / /$ (hace un movimiento
circular con la mano derecha)
CUÁNTO DOLOR:: $\uparrow / /$ (eleva la mano
izquierda hacia arriba)
TODOS murieron $\downarrow / /$
Mi madre murió allí/ en nuestra casa//
(señala con la mano izquierda hacia atrás
con un movimiento circular y girando
levemente el cuerpo)
QUÉ DOLOR:: $\uparrow / /$ (se lleva la mano al
pecho) [...]

$[\ldots]>$ TODOS nuestros antepasados ya murieron $<\rightarrow / /$ (hace un movimiento circular con la mano derecha) CUÁNTO DOLOR:: $\uparrow / /$ (eleva la mano izquierda hacia arriba) TODOS murieron $\downarrow / /$

Mi madre murió allí/ en nuestra casa// con un movimiento circular y girando levemente el cuerpo) pecho) $[\ldots]$

Junto a estos conceptos existe otro que se vincula más bien con la desazón que produce la carencia material: paravete. Así: mbaeti anoi, che paravete 'no tengo nada, soy carente'; iparavete uiko 'andan con carencias'. En algunos casos, los chanés desconfían de ello diciendo, por ejemplo, jae iparavete iyeyapo ' finge que tiene carencias'. Branislava Susnik nota que, entre los chiriguanos -emparentados histórica, cultural y lingüísticamente con los chanés- el peor insulto posible era tinei o 'huérfano', justamente por la estrecha asociación con la categoría social de paravete. Esto es porque la falta de lazos sociales lleva aparejada la escasez de recursos materiales importantes; es decir -y esto todavía puede observarse entre los chanés-, que una persona que no goza de lo que Susnik (1968, p. 12) denomina "solidaridad obligatoria de los parientes" tendrá posibilidades muy limitadas de realizar un convite de cualquier tipo por falta de alimentos y bebida para ofrecer, y quedaría entonces por fuera del circuito del umatiro o 'trabajo colectivo'. Para ejemplificarlo tomaré un fragmento de un relato que registré en 2018 en el que una mujer llamada Juana, de en ese entonces 51 ańos, describe el modo en el que durante la Guerra del Chaco (1932-1935) librada entre Paraguay y Bolivia muchos indígenas migraban hacia la Argentina (Bossert, Combès y Villar, 2008): 
JOKUAE KARAMBOE个//

Guerra del Chacope $\downarrow / /$

JETA MBAA JARE KUNA KOTH/

Boliviagui/ ou $\rightarrow / /$ (señala hacia atrás con la mano derecha)

Kuña ime ndie ou $\rightarrow / /$

JOKUA MBAA POCHI GUI NDAYE $\rightarrow / /$

ARAKAE UYERERURETA KUARAI

$P A R A V E T E \rightarrow / /$

Kuae tape ou Mbaaporendape $\rightarrow / /$

Jama arakae uyereru-reruretal $\rightarrow$ I

JAMA KOROPI:: oo kuña imendie $\uparrow / /$

(extiende la mano derecha y la mueve

lentamente de un lado al otro)

I opa chuguireta $\rightarrow / /$

JAMA $\mathrm{I}$ OPA $\rightarrow / /$

Jama jokuae kuña uvapi ivipei $\rightarrow / /$

Ipuereama uvata $\downarrow / /$

IPUEREAMA $\downarrow / /$ (niega con la cabeza)

Ikandi $\rightarrow / /[\ldots]$
ASÍ ERA HACE MUCHO

TIEMPO $\uparrow / /$

En la época de la Guerra del Chaco $\downarrow / /$

MUCHOS HOMBRES Y

MUJERES DE ALLÍ/ de Bolivia/

venían $\rightarrow / /$ (señala hacia atrás

con la mano derecha)

Venían una mujer y su marido $\rightarrow / /$

EL HOMBRE SE HABÍA

ENOJADO CON SU MUJER

SE CUENTA $\rightarrow / /$

ASÍ VENÍAN ANTIGUAMENTE

POBRECITOS $\rightarrow / /$

Venían por ese camino que venía a

Argentina $\rightarrow / /$

Antiguamente venían seguido $\rightarrow / /$

Y POR ALLÍ:: iban la mujer y su marido $\uparrow / /$

(extiende la mano derecha y la mueve

lentamente de un lado al otro)

El agua se les terminó $\rightarrow / /$

SE TERMINÓ EL AGUA $\rightarrow / /$

Y la mujer se sentó en la tierra $\rightarrow / /$

Ya no podía caminar $\downarrow / /$

YA NO PODÍA $\downarrow / /$ (niega con la cabeza)

Estaba cansada $\rightarrow / /[\ldots]$

En la anteúltima línea del relato se puede observar de qué forma la narradora utiliza el vocablo paravete en sentido amplio, para referirse a las condiciones en las que los migrantes indígenas transitaban el camino que los llevaba a esta nueva tierra: a pie, sin demasiados víveres y enfrentándose a distintos tipos de peligros.

Por el contrario, lo relacionado con el 'gozo', el 'contento' y la 'alegría' o la 'diversión' cae bajo la categoría de yerovia. Obsérvese los siguientes ejemplos: tuicha ayerovia 'estoy muy alegre'; yayerovia arete guasu ou ramo 'estamos alegres porque ha llegado el carnaval'; kuña yeroviambae 'mujer sin alegría'. Más allá de sus sentidos originales, esta palabra ha sido utilizada por los misioneros como traducción para el concepto de 'fe' cristiana o también de 'creencia en Dios' (Morando, 2017). Un sinónimo de yerovia es tori. Tori está vinculado con la 'alegría' aunque, al contrario del primero, no parece haber adquirido con el paso del tiempo un sentido religioso, tal como se puede apreciar en los siguientes ejemplos: che rori ndere 'estoy contento/a por ti'; chemembi uiko rori 'mi hijo/a anda contento'; kuae kuña jori 'esa mujer está contenta'; yapirae rori 'cantamos contentos'.

Contrariamente, mbotarei se relaciona con los sentimientos de 'aborrecimiento', 'rechazo' y 'animosidad'. Así: cheme chembotarei 'mi marido me detesta'; aguiye chembotarei 'no me detestes'; che tuicha romotarei 'te detesto mucho'.

Además, puede mencionarse la palabra pirerekua, que los chanés suelen traducir como 'generosidad', 'bondad' e inclusive 'compasión'. Esta es, por ejemplo, una de las características fundamentales de la autoridad del mburuvicha o 'cacique' (Villar, 2005, p. 31)-; así, es posible 
escucharla utilizada en frases como umee ñandeve, ipirerekua 'nos da a todos, es generoso'; ipirerekua yeye 'es muy generoso'; mburuvicha tuicha ipirerekua 'el cacique es muy generoso'. Compárecelas con otros ejemplos similares relevados por Villar (2005, p. 31) como: ketipa rojota roeka mbororekua? '¿dónde encontraremos alguien que nos compadezca?'; mburuvicha upirerekua ka 'el cacique es generoso'.

Por último, haré una breve mención al vocablo aiu, que podría traducirse como 'amor' o 'aprecio'. Se puede escuchar utilizada en expresiones como: che aiu chimbireko 'yo amo a mi esposa', o bien kuae sambiaireta uaiu ichi 'esos niños aman a su madre'. Alternativamente, los chanés también suelen utilizar con significado similar el verbo pota 'querer' en expresiones como: che tuicha nduipota 'te quiero mucho'; aipotama ndere 'ya te estoy queriendo'.

\section{Palabras finales}

Pretendí esbozar aquí un acercamiento etnográfico a la construcción de la emoción desde lo discursivo entre los chanés del Noroeste Argentino recuperando aquellas expresiones por medio de las cuales se expresan los estados anímicos, haciendo particular hincapié en la categoría de $p \dot{t} a$. Del análisis podemos desprender que el $p \dot{t} a$ tiene al menos dos facetas. Por un lado, revela un evidente anclaje orgánico que le permite canalizar expresivamente procesos fisiológicos como los dolores abdominales, los ritmos cambiantes de la frecuencia cardíaca, las convulsiones o los temblores. Pero, por otro, el pia también opera encarnando y dando forma material a una gama variada de emociones y conductas; puede ser 'frío' si una persona se encuentra sosegada, 'grande' si se trata de una persona 'arrogante' y 'orgullosa', o incluso puede asumir cierta agencia sintiendo 'miedo', 'llorando' o incluso 'muriendo' frente a una sorpresa grande. Se trata de una noción polisémica con al menos dos caras de sentido que deben ser integradas y tomadas en conjunto para comprender más acabadamente lo que representa implícita y explícitamente para los hablantes y, por ende, su poder evocador de lo emotivo en el contexto de la experiencia social.

La existencia de ambas facetas plantea ciertamente una suerte de tensión entre la vida interior de la persona y el contexto social externo (Descola, 1996 [1987]; Surrallés, 2009 [2003]), que sin embargo no resultan irreconciliables entre sí, sino que constituyen elementos funcionales de gran importancia a la hora de expresar las emociones. Dada esa doble naturaleza, podría decirse que esta noción asume casi la totalidad de la persona o del "yo" chané. Así, el pia se constituye como un vector de subjetividad en la configuración anímica de la persona y detenta también un lugar central en la matriz de significados compartidos en la vida social chané al operar constituyendo, estableciendo y reafirmando las relaciones interpersonales (Seeger, Da Matta y Viveiros de Castro, 1979). Coincido en este sentido entonces con la tesis planteada por Graciela Chamorro desde el caso de grupos guaraníes orientales (2009, p. 152), para quien el posible significado primario del término podría interpretarse como el "centro de la vitalidad humana" y cuyo sentido ulterior es plausible de ser comprendido según el contexto discursivo.

El análisis nos coloca además frente a una cuestión importante desde el punto de vista de la traducción. Encontrar una traducción verbatim para las categorías indígenas resulta en muchas ocasiones una tarea sumamente compleja, infértil y, en cierto modo, hasta innecesaria. En efecto, lo más frecuente es que la realidad etnográfica exceda cualquier tipo de matiz de traducción (Needham, 1972). Por ello, más que una adición progresiva de significados a la manera de un catálogo, lo que pretendí aquí fue tensionar los límites que plantea la labor de traducción intercultural para poder abordarla como proceso hermenéutico continuo y flexible, cuyo foco 
sea la interpretación de signos lingüísticos que permita acceder a los significados de la palabra indígena en su sentido más conceptual.

\section{Agradecimientos}

Agradezco al Dr. Diego Villar por sus valiosos aportes a las versiones preliminares de este trabajo, así como también a los evaluadores anónimos y al equipo editorial de la revista Estudios Atacameños. Arqueología y Antropología Surandinas por la exhaustiva revisión de las informaciones aquí consignadas. Reconozco también a todos aquellos que me acogieron desinteresadamente y me brindaron su apoyo en las comunidades en las que he realizado trabajo de campo desde el ańo 2012. La investigación de la cual han sido producto los datos presentados aquí fue financiada por medio de una beca interna doctoral del Consejo Nacional de Investigaciones Científicas y Técnicas (CONICET) de Argentina

\section{Referencias citadas}

Aikhenvald, A. (1999). The Arawak language family. En Dixon R. y Aikhenvald A. (Eds.). The Amazonian languages (pp. 65-106). Cambridge: Cambridge University Press.

Bauman, R. (1975). Verbal art as performance. American Anthropologist, 77(2), 290-311.

Bauman, R. (1977). Verbal art as performance. Illinois: Waveland Press.

Bauman, R. (1986). Story, performance and event. Contextual studies of oral narrative. Cambridge: Cambridge University Press.

Bauman, R. y Briggs, C. (1990). Poetics and Performance as Critical Perspectives on Language and Social Life. Annual Review of Anthropology, 19, 59-88.

Bossert, F., Combès, I. y Villar, D. (2008). La guerra del Chaco entre los chané e isoseños del Chaco occidental. En Richard, N. (Ed.). Mala guerra. Los indigenas en la guerra del Chaco (1932-1935) (pp. 203-233). Asunción: Museo del Barro.

Chamorro, G. (2009). Decir el cuerpo. Historia y etnografía del cuerpo en los pueblos guarani. Asunción: Tiempo de Historia/ FONDEC.

Combès, I. (2005). Etno-historias del Isoso. Chané y chiriguanos en el Chaco boliviano (siglos XVI-XX). La Paz: IFEA.

Combès, I. (2007). De Sanandita al Itiyuro: los chanés, los chiriguanos (¿y los tapietes?) al sur del Pilcomayo. Indiana, 24, 259-289.

Combès, I. y Saignes, T. (1995). Chiri-guana: nacimiento de una identidad mestiza. En Riester, J. (Ed.). Chiriguano: Pueblos indigenas de las tierras bajas de Bolivia (pp. 25-221). Santa Cruz de la Sierra: APCOB.

Combès, I. y Villar D. (2004). Aristocracias chané. "Casas" en el Chaco argentino y boliviano. Journal de la Société des Américanistes, 90(2), 63-102.

Córdoba, L. (2006). Idéologie, symbolisme et rapports entre sexes dans la construction de la personne chacobo. Recherches Amérindienne au Québec, 36(1), 59-68. 
Córdoba, L. (2008). Parentesco en femenino: género, alianza y organización social entre los chacobo de la Amazonía boliviana. Tesis de Doctorado, Universidad de Buenos Aires, Argentina.

Corrado, A. M. (1871). Catecismo de la Doctrina Cristiana con varias oraciones y prácticas devotas en lengua Chiriguana con su traducción literal al castellano. Sucre: Imprenta de Pedro España.

Cuneo, P. y Messineo, C. (Inédito). My heart laughs. Patterns of Lexicalization of Body-Part Terms and the Expression of Emotion in Toba/Qom (Guaycuruan, Gran Chaco Region. En Zariquiey, R. y Valenzuela, P. (Eds). The Grammar of Body-Part Expressions in Amerindian Languages.

Da Matta, R. (1976). Um mundo dividido: a estrutura social dos indios Apinayé. Petrópolis: Vozes.

Descola, P. (1996 [1987]). La selva culta: simbolismo y praxis en la ecología de los Achuar. Quito: Abya-Yala.

Dietrich, W. (1986). El idioma chiriguano: Gramática, textos, vocabulario. Madrid: Ediciones Cultura Hispánica.

Giannecchini, D. (1869). Carta del P. Doroteo Giannecchini al R.P. guardián y venerable discretorio, 26.VI.1869. Tarija: Archivo Franciscano de Tarija [AFT 1-878].

Giannecchini, D. (1916 [1896]). Diccionario Chiriguano-Español y Español-Chiriguano. Tarija: Publicación de la Orden Franciscana.

Golluscio, L. (2019 [2002]). Etnografía del habla. Textos fundacionales. Buenos Aires: EUDEBA.

Golte, J. (1973). El concepto de sonqo en el runa simi del siglo XVI. Indiana, 1, 213-218.

Gómez, C. (2010). La luna y la feminidad entre los tobas del oeste formoseño (Gran Chaco, Argentina). Campos, 11(1), 47-64.

Gumperz, J. (1982). Discourse strategies. Cambridge: Cambridge University Press.

Gustafson, B. (2014). Guaraní. En Crevels, M. y Muysken, P. (Eds.). Lenguas de Bolivia. Tomo 3. Oriente (pp. 307-368). La Paz: Plural Editores.

Henry, J. (1936). The linguistic expression of emotion. American Anthropologist, 38(2), 250-256.

Hymes, D. (1981). "In Vain I Tried to Tell You": Essays in Native American Ethnopoetics. Filadelfia, PA: University of Pennsylvania Press.

INDEC (2004/2005). Encuesta Complementaria de Pueblos Indígenas (ECPI) -Complementaria del Censo Nacional de Población, Hogares y Viviendas 2001. Buenos Aires: Instituto Nacional de Estadística y Censos de la República Argentina.

Langer, E. (1995). Misiones franciscanas y trabajadores chiriguanos: colonización, aculturación y mano de obra indígena en el sudeste de Bolivia. En Riester, J. (Ed.). Chiriguano: Pueblos indígenas de las tierras bajas de Bolivia (pp. 255-287). Santa Cruz de la Sierra: APCOB.

Leenhardt, M. (1997 [1947]). Do Kamo: la persona y el mito en el mundo melanesio. Buenos Aires: Paidós.

León de Santiago, P. (1791). Diccionario Breve Chiriguanae. Tarija: Archivo Franciscano de Tarija. AFT-11835/MS16.

Magrassi, G. (1968). El complejo chiriguano-chané. En Ministerio del Interior. Censo Indigena Nacional, Vol. 2 (Provincias de Chaco, Formosa, Jujuy, Misiones, Salta y Santa Fe) (pp. 23-60). Buenos Aires: Ministerio del Interior. 
Métraux, A. (1930). Études sur la civilisation des indiens Chiriguano. Revista del Instituto de Etnología de la Universidad de Tucumán, 1, 295-493.

Métraux, A. (1948). Tribes of the Eastern Slopes of the Bolivian Andes. Chiriguano and Chané. En Steward, J. (Ed.). Handbook of South American Indians, Vol. 3 (pp. 465-485). Washington, DC: Smithsonian Institution.

Montani, R. (2012). La construcción material de la persona entre los wichís del gran chaco. Avá, 22, 167-190.

Montani, R. (2017). El mundo de las cosas entre los wichis del Gran Chaco. Un estudio etnolingüistico. Cochabamba: ILAMIS/ Itinerarios.

Morando, M. A. (2015). Bilingüismo y organización social en la comunidad chané de Tuyunti (departamento General San Martín, provincia de Salta). Suplemento Antropológico, 50(2), 257-335.

Morando, M. A. (2017). Visiones de lo religioso entre los chiriguano: la lexicografía franciscana entre los siglos XVIII y XIX. Anuario de Estudios Bolivianos, Archivisticos y Bibliográficos, 23(1), 199-221.

Needham, R. (1972). Belief, Language, and Experience. Oxford: Basil Backwell.

Overing, J. (1977). Orientation for paper topics and comments: Symposium Social Time and Social Space in Lowland South American Societies. Actas del XLII Congreso International de los Americanistas, 2(9-10), $387-94$.

Palavecino, E. (1949). Algunas informaciones de introducción a un estudio sobre los chané. Revista del Museo de La Plata, 4, 117-131.

Palmer, J. (2005). La buena voluntad wichí. Una espiritualidad indígena. Formosa/Salta: Grupo de Trabajo Ruta 81.

Rocca, M. (1973). Los Chiriguano-Chane. América Indigena, 33(3), 743-756.

Rocca, M. y Newbery, S. (1972). El carnaval chiriguano-chané. Cuadernos del Instituto Nacional de Antropología, 8, 43-91.

Saignes, T. (2007). Historia del pueblo chiriguano. La Paz: IFEA/Plural.

Seeger, A., Da Matta R. y Viveiros de Castro E. (1979). A construção da pessoa nas sociedades indígenas brasileiras. Boletim do Museu Nacional, 32, 2-19.

Sherzer, J. (1987). A Discourse-Centered Approach to Language and Culture. American Anthropologist New Series, 89(2), 295-309.

Surrallés, A. (2009 [2003]). En el corazón del sentido. Percepción, afectividad, acción en los candoshi, Alta Amazonía. Lima: IFEA.

Susnik, B. (1968). Chiriguanos I. Dimensiones etnosociales. Asunción: Museo etnográfico Andrés Barbero.

Tola, F. (2007). Constitución de la persona sexuada entre los toba, qom, del Chaco argentino. Revista pueblos y fronteras digital, 2(4), en línea: http://dx.doi.org/10.22201/cimsur.18704115e.2007.4.227.

Vilaça, A. (2005). Chronically unstable bodies: reflections on Amazonian corporalities. The Royal Anthropological Institute, 11(3), 445-464. 
Villar, D. (2005). La religión chané. Tesis de Doctorado, Universidad de Buenos Aires.

Villar, D. (2006). Repensando el complejo cultural chiriguano-chané. En Combès, I. (Ed.). Definiciones étnicas, organización social y estrategias políticas en el Chaco y la Chiquitania (pp. 205-224). Santa Cruz de la Sierra/Lima: IFEA / SNV/ El País.

Villar, D. (2008). Guaranización, traducción y evangelización en las representaciones anímicas chané. Suplemento Antropológico, 43 (1), 339-386.

Villar, D. (2011). La religión del monte entre los chané. Suplemento Antropológico, 46(1), 151-201.

Villar, D. y Bossert, F. (2014). Máscaras y muertos entre los chané. Separata, 19, 12-33.

Viveiros de Castro, E. (1996). Os pronomes cosmológicos e o perspectivismo ameríndio. Mana, 2(2), 115143. 